\title{
Civil Identification as a Unique Patient Identifier for Hospitals in
}

\section{Kuwait}

\author{
Kamel A. Alsaleh*, Faisal Al-sharifi ${ }^{\star \star}$, Bader Al-Khalaf $^{\dagger}$, Samy Elewa $^{\ddagger}$
}

\begin{abstract}
Background: A unified patient identifier is an essential item in improving the quality of health care delivery. This is the trend and major progress has been achieved in many developed and developing countries. In Kuwait each hospital had its own identifier and linking the patient data is rather difficult. The Public Authority for Civil Identification (PACl) Civil Identification number (CID) is the most promising candidate and all the hospitals have been instructed and persuaded to include the CID in the discharge forms, this was stressed since 1995. Objective: The aim of the study is to find out the availability of the PACI ICD on the hospital discharge forms. Methods: The data-bases storing hospital discharge data for the government hospitals were reviewed for the presence of CID number during the period 19962005. A retrospective approach using the discharge summary data-base of the Statistical and Medical Record Department, MOH, Kuwait was used. Results: The rate of inclusion of the CID was very small in 1996 (13.6\%.) increased in 1997 to $30.2 \%$ and, remained approximately at that level until 2002. Since 2003 the rates increased substantially and amounted in 2005 to $68.1 \%$. Marked variability was seen between the hospitals, Sabah and Ibn Sina were the least reporting, being around $7 \%$, Mobarak and the Chest Hospital scored above $66 \%$. Getting a unified patient identifier is not a goal by itself; it is a step on the road to improve the health services. A major advantage, which was not addressed in this survey, is the inclusion of the CID in the Death certificate. This will provide the potential of linking the 2 data bases at a later stage. Conclusions: PACI CID includes the basic information that can make it an appropriate deterministic identifier. The response of the hospitals is favorable and the system should be field tested.
\end{abstract}

Kew words: Kuwait, hospital ID, Civil CID, PACI

\section{INTRODUCTION}

Patient identifiers are vital for day-to-day

operation of health care facilities; this

includes the delivery of care, the availability of complete health care administrative processes, support information on which current and future

Department of Medical Records, College of Health Sciences, The Public Authority for Applied Education and Training, State of Kuwait

¿Department of Environmental Health, College of Health Sciences, The Public Authority for Applied Education and Training, State of Kuwait

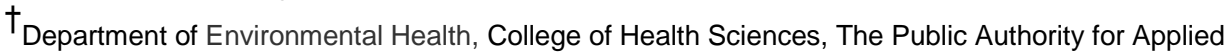
Education and Training, State of Kuwait

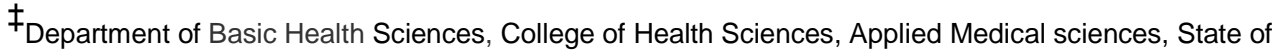
Kuwait 
care can be planned and implemented. Patients, however, may have multiple records - even within a single institution often unknown by the institution or the individual care provider.(2) Today, one of the main objectives of the interfaced health information systems or networks is to be able to gather the different parts of the medical record of a patient without any risk of mixing them with those of another patient. This objective could appear easy to reach, but only in theory because in practice many names are misspelled or erroneous. Great attention has to be paid to define the best identifiers that would be used to link medical records. A linkage using less informative identifiers could lead to linkage errors: it is essential to quantify the information associated to each identifier. $^{3}$ There are 2 commonly used combinations of four personal identifiers computerized record linkage approaches: namely, the deterministic and probalistic. ${ }^{(4)}$ The deterministic approach generates links on the basis of a full agreement of a unique identifier or a set of common identifiers. This method minimizes the uncertainties in the match between 2 databases at the cost of lowering the linkage rate. The probabilistic record linkage approach links 2 data bases based on calculated statistical probability of a set of common identifiers. The probability is used to determine whether a pair of records approximately refers to the same individual. This would maximize linkage theoretically but may result in uncertainty for some potential links. In all cases, it seems necessary to improve the quality of identifier collection as it could greatly influence linkage results. ${ }^{(3)} \quad$ The 
were compared, the combination of the quality of the underlying data. ${ }^{(8)}$

surname, sex, and date of birth had the highest linkage rate of $88.0 \%$ and $93.1 \%$ adding the first name to the combination increased correct linkage by less than $1 \%$, but at the cost of lowering the linkage rate almost by $10 \% .^{(5)}$

Health services are undergoing a large paradigm change: a shift from institutionCentered to patient-centered care. ${ }^{(6)}$ Enterprise Master Patient Indexes (EMPIs) can serve as critical tools in improving operations, protecting medical record integrity, and improving patient service. ${ }^{(7)}$ Historically, the health information systems community has viewed linking personal records as an easy task. The oversimplified view that routine database manipulation can accurately identify multiple records for a single individual is erroneous, an assumption based on a misperception of
The ability to share patient specific health data is essential to provision of care and facilitate research in biomedical information: at the same time it is necessary to uphold patient privacy rights. ${ }^{(9)}$ The Privacy Rule of the Health Insurance Probability and Accountability Act $\left({ }^{10)}\right.$ in the USA requires data holders to render personal health information anonymous. Until recently anonymity was assumed when data was stripped of explicit identifying information: however, de-identification methods do not guarantee the anonymity of health data.(11). A novel computational protocol that enables data holders to work together to determine records that can be disclosed and satisfy a formal privacy protection was described. ${ }^{(9)}$

The current method of patient identification in Kuwait is based on the 
use of a medical record number, issued and maintained by the provider organization. This number is based on an institutional Master Patient Index (MPI), and the numbering system is specific to the organization. Different providers use different numbering systems: the same patient often receives several Medical Record Numbers, unique to the organizations. These numbers provide identification within the individual organization. Collection of data on hospital discharged patients was started in Kuwait during 1985 and has been managed since by the Vital and Health Statistics Section. Information from the medical record is recorded on an abstract form by hospital medical record department staff.

Looking for a patient identifier for Kuwait was a lengthy endeavor that information includes the nationality, sex, started since some years. Lengthy the Ministry of Interior residency number discussions between $\mathrm{MOH}$, Ministry of Planning (MOP), Ministry of Interior (MOI), and Public Authority for Civil Identification (PACl) were made. The civil identification (CID) organized and maintained by $\mathrm{PACl}$ was considered the most appropriate candidate on which to build the patient identification. It is an established system of high credibility and was running for years. The system is based on allocating a 12 digit unique number to each resident in Kuwait whether a national or a non-Kuwaiti. PACI CID numbering system is based on the date of birth in Century (1800-1899 $=1),(1900-1999=2)$, and (2000-2099 $=3$ ). The year, month, and day of birth are given 2 digits each. A CID number is given another 4 digits and a check digit is added to the number. Additional 
for the non-nationals, home address, telephone number and blood group. For the nationals, the same number is kept until death and will never be used again. For non-nationals it is linked to the legal length of residency and suspended if the person would leave the country. On coming back the individual will get the same number. The civil ID is a recognized and accepted document, it should be updated regularly and there is a fine if it is not renewed in time. It is accepted as a document and is used in the banks, or in any encounter with any official organization. Great pressure was exerted by the Ministry of Health to include the civil ID in the discharge abstract sheets. This was started early in 1990s, however, the response was not good, a serious attempt was made in 1995 and it came with the $\mathrm{MOH}$ decision to collect certain nominal fees for certain services from certain groups of the nonnationals.

Objective: The study was designed to determine the rate of inclusion of the CID in the discharge abstract forms of the government hospitals.

\section{Methods}

The study employed a retrospective approach using the discharge summary data base of the Statistical and Medical Record department, MOH, Kuwait. Using a special algorithm all discharge abstracts from the government hospitals available at the data base were reviewed for the presence of Civil ID. There was no available recorded data regarding Civil ID before 1996 in the data base. The frequency of including the CID in the hospital discharge was sought through an extensive review of the hospital discharge abstracts in the 10 years 1996 to 2005 . 


\section{RESULTS}

The availability of the CID according to the year for the government hospitals is summarized in table 1 . The rate of inclusion was very low at the start of the period $(13.6 \%)$ in 1996. A steady rise was seen all through the period. The proportion of the cases in which the CID was included in 1997 amounted to $30.2 \%$. This was more than double that for 1996. The rate of reporting was similar starting in 1997; being slightly over $30 \%$ in all the years up to 2001 . A distinct rise was seen in 2002 and the proportion of inclusion amounted to $42.8 \%$, in 2003 the rate was $56.2 \%$, it came up to $59.8 \%$ in 2004 and was somewhat higher in 2005 (68.1\%). This was almost 5 times that for 1996, moreover, it represent two-thirds of the discharges.
The inclusion rate according to the hospital (Table 2) revealed much more variability. The availability of ID was very low for Sabah $(7.1 \%)$ and Ibn Sina (7.5\%): the first is a major general hospital and the second is a specialty hospital providing tertiary care for neurology, ophthalmology, neurosurgery and various other specialties. A much higher rate was reported for Mobarak (66.3\%), Chest Hospital (66.2\%) and the Maternity Hospital (74.2\%). There was no consistent pattern for the general hospitals, Farwania had a rate of inclusion of $51.6 \%$, this was higher than any other general hospital. The rates for Adan (22.9\%) and Jahra (26.7\%) were close, both were almost $50 \%$ of the rate of inclusion for Farwania.

Marked variability was also in the frequency of reported data for each hospital in the different years. The 
results are shown in table 3 . For Amiri Hospital, the proportions were less than $5 \%$ until 2001, the situation improved markedly after that. With Sabah Hospital, the proportion was less than $10 \%$ for the period 1996-2002, the overall reporting was low and was around 25\% for 2005 . Reporting was better for Farwania, Adan, the Infectious Diseases, and Chest Diseases hospitals. Data for the Allergy Hospital was only reported for 2005 . The numbers reported for the Physical Medicine Hospital were quite small. No data was reported by Sabah Hospital in 1998, and Ibn Sina. The Chest Diseases Hospital failed to report in 2003.

\section{DISCUSSION}

The increased availability of large scale health care data sets has improved the ability to assess the quality of health care delivery. Linking data sources allows for richer and more revealing analysis of health care. ${ }^{(12,13)}$ The use of PACI CID was based on the assumption that it would be a way out, the CID has the essential information thought to uniquely identify the subject. It is based on the inclusion of the birth date and the name, these are the major items recommended by many scholars in the

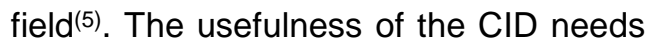
to be verified for validity, and tested for accuracy. The system was initiated keeping in mind assigning a unique identifier for the people residing in Kuwait. Though the system did not have the hospitals or patient identity in mind at its conception, yet the availability would be very beneficial to the health services. Moreover, the use of the system should be considered very cost effective and the merits would be exploited and validated regularly in the years to come. 
In recent years there has been a hospitalization to death records is tremendous need among health care already performed usually on an as professionals to assess the needed basis for research purposes. A effectiveness, efficiency, and significant weakness of many data appropriateness of the patient care linkage projects and subsequent use of services being provided through criteria- such data is lack of knowledge regarding based outcome and program evaluation. the magnitude of potential bias due to Although the need for a tool which could missing data.(13) It is worth noting that the evaluate the effectiveness of patient care CID in Kuwait is included in the death is widely recognized, such an certificate. A study to assess the undertaking has been severely limited feasibility to combine the data from due to the lack of any automated means hospital discharge with the cause of to collect and analyze patient data on a death on the death certificate would be a routine, continuous basis within a clinical step in the right direction.

setting. ${ }^{(14)}$ Having a unified patient The utilization of the CID would not identification is only a step on the way, be limited to hospital or other health care the system management should facility day-to-day operation, it would incorporate expert rules, dynamic data extend to the post hospital period. This entry forms, quantitative models, and includes provision of rehabilitation care. user-defined access control. This is essential to prevention of

Mortality is an accepted measure of disability, to promote patient autonomy the quality of health care. Linkage of and to avert the need for long-term 
care $^{(14)}$. The home care is also relevant in view of the trend that more people with terminal conditions prefer to stay at home. ${ }^{(15)}$

The developed system produces patient-specific reports with many dimensions. It provides the framework for continually capturing data at a practical, work-flow level. The incorporation of a dynamic patient database as the driving force of an integrated, rule-based administration, financial and patient data system will provide the tools for health care. ${ }^{(14)}$ The system would check up on several other health components like vital statistics. Considerable discrepancy was found between death certificate and corresponding hospital discharge records. It was found that the underlying cause of death and the main condition differed at basic tabulation list level in
$54 \%$ of the deaths. One-third of the differences could not be explained by ICD selection procedures. ${ }^{(16)}$ Adding hospital discharge data changed the underlying cause in $11 \%$ of the deaths, and for some causes the effect was substantial. ${ }^{(16)}$

An integrated electronic patient record (EPR) was developed by the hospital authority in Hong Kong to meet the high tempo health care environment; the model was further developed to extend the longitudinal EPR to include fetal data as entered in the mother record, the intention was to build a true womb-to-tomb EPR for the patient.(17) Further development was described. This was to implement a web based electronic patient record system to allow integrated real time patient based information to be shared in clinics, private, and public hospitals; such 
sharing aims at insuring that complete and accurate healthcare information is available to citizens' multiple points of care through system. The challenge is to share this information whilst ensuring privacy and security. It was also noted that Hong Kong already achieved its initial goal and pioneered building a territory wide Electronic Health Record (HER). ${ }^{(18)}$

$\mathrm{PACl}$ record number is a deterministic indicator. The next step is to apply a linkage algorithm. Several algorithms are available commercially and $\mathrm{MOH}$ would pick up the most appropriate. Testing the accuracy of each would be undertaken; sensitivity and positive predictive value ${ }^{(19)}$ would be followed. Though it is a deterministic indicator, the accuracy may be compromised by wrong entry of data, thus the PACl number should be supplemented with other demographic indicators.

\section{CONCLUSION AND RECOMMENDATIONS}

It could be concluded that there was marked improvement in the rate of inclusion of the civil ID in the study period; however several hospitals were still lagging behind. These include one general hospital (Sabah) and Ibn Sina a member of the specialty hospitals. The progress in inclusion is encouraging but the persistence is an item that would be followed-up. The progress in the inclusion after 1995 points to the decisive role of the hospital administration in enforcing the rules that have been issued by $\mathrm{MOH}$. The clerks (Receptionist and Abstractors) should be educated about the importance of the inclusion of the CID, persuaded, and supervised. Every body has a CID and 
the receptionist would ask for it and should be explained to the medical record in any encounter

record departments within the hospitals

Improvement in data quality should and a sense of pride in the job impeded. be an on-going issue for data collection The technicians should be aware of the Training of the technicians feeding in the importance of their work. The use of a data should be considered the corner unified CID is a tool of very high stone for any progress, they should be potential, the example of Hong Kong is persuaded and close supervision should eye opening, a similar system would be be provided. The importance of the data eventually sought. 
Table 1: Availability of CID according to the Year (1996-2005)

\begin{tabular}{|c|c|c|}
\hline Year & No. of discharge abstracts & \% Abstracts including CID \\
1996 & 23960 & 13.6 \\
\hline 1997 & 53000 & 30.2 \\
\hline 1998 & 52948 & 33.7 \\
\hline 1999 & 55592 & 31.8 \\
\hline 2000 & 64513 & 35.4 \\
\hline 2001 & 62877 & 34.1 \\
\hline 2002 & 79868 & 42.8 \\
\hline 2003 & 91318 & 56.2 \\
\hline 2004 & 109131 & 59.8 \\
\hline 2005 & 129033 & 68.1 \\
\hline
\end{tabular}

Table 2: Availability of CID on Patient discharge abstracts according to the Hospital type

\begin{tabular}{|l|c|c|}
\hline \multicolumn{1}{|c|}{ Hospital } & $\begin{array}{c}\text { No. of discharge } \\
\text { abstracts }\end{array}$ & $\begin{array}{c}\text { \% Abstracts } \\
\text { including CID }\end{array}$ \\
\hline General Hospitals: & & \\
\hline Amiri & 44156 & 34.6 \\
\hline Sabah & 13291 & 7.1 \\
\hline Mobarak & 126342 & 66.3 \\
\hline Farwania & 67596 & 51.6 \\
\hline Adan & 77821 & 22.9 \\
\hline Jahra & 77821 & 26.7 \\
\hline & & \\
Specialized Hospitals: & 32941 & 66.2 \\
Chest & 161421 & 74.2 \\
Maternity & 10483 & 40.8 \\
Psychiatric & 10944 & 58.3 \\
KCCC & 29369 & 54.4 \\
Al-Razi & 2811 & 79.5 \\
Physical Medicine & 23670 & 63.4 \\
Infections & & \\
\hline
\end{tabular}




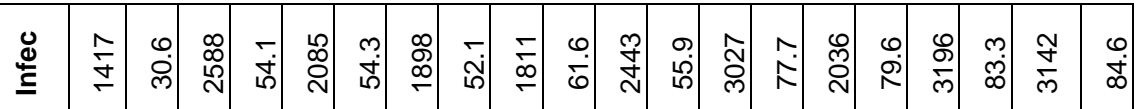

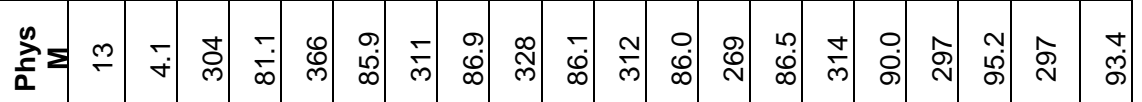

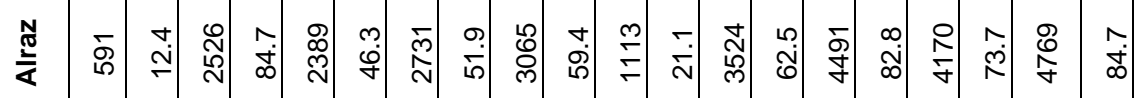

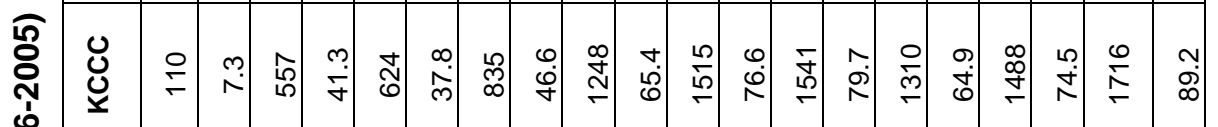
ڤั

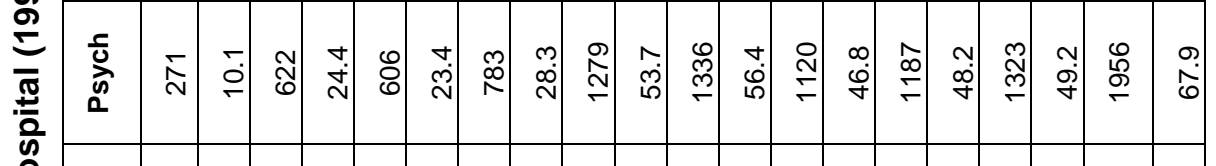

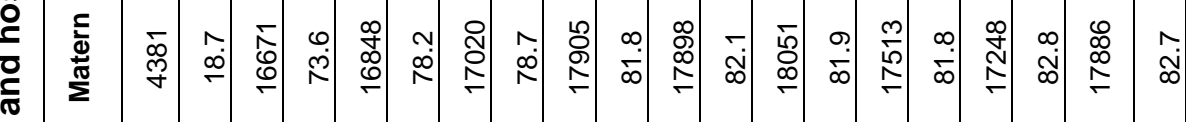

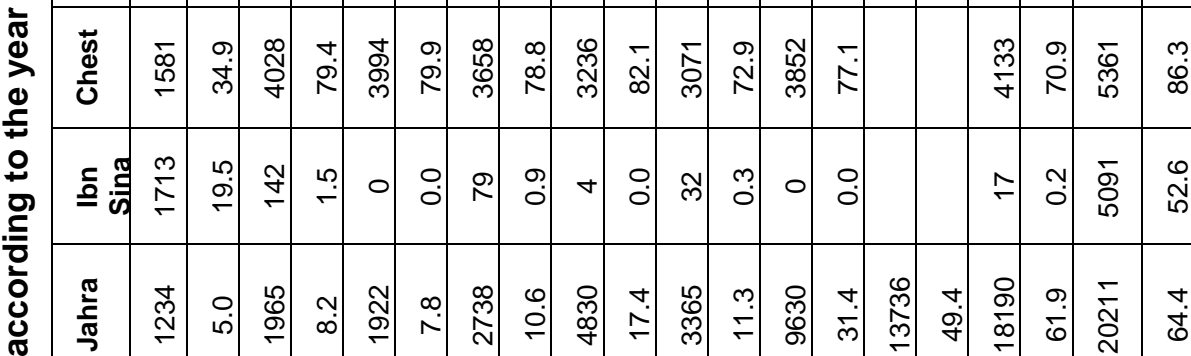
믈

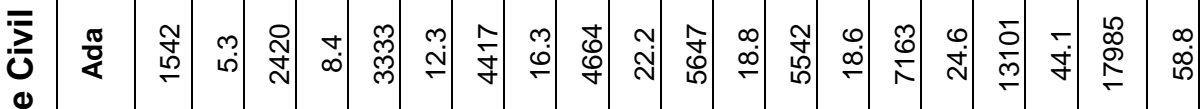

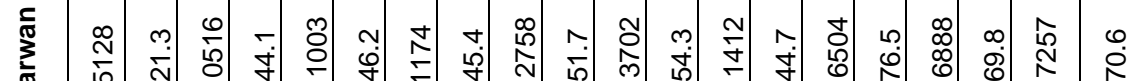
ป

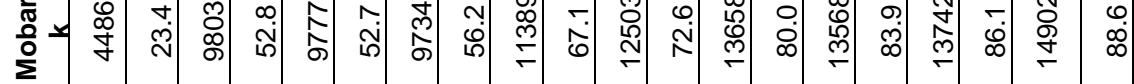

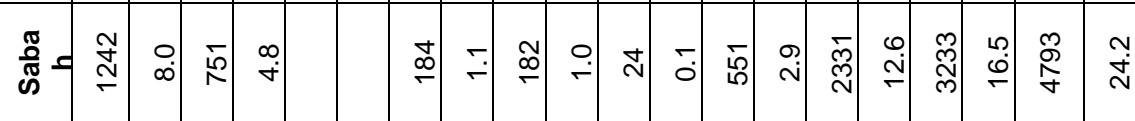

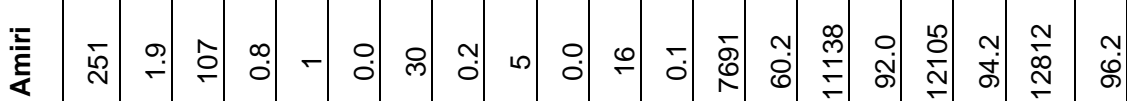

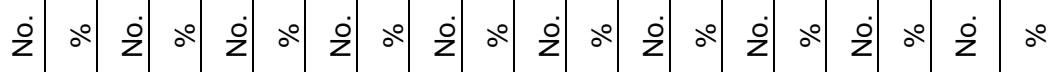

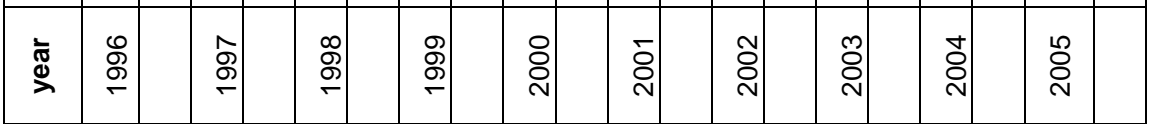




\section{REFERENCES}

1. Mills ME. Linkage of patient records to support continuity of care: Issues and future directions. Stud Health Technology Inform. 2006; 122:3204.

2. Brook EL, Rosman DL, Holman CDJ, Trutwein B. Summary report research output project, WA data Linkage Unit (1995-2003). Perth: Department of Health Western Australia; 2005.

3. Quantin C, Binquet C, Bourquard K, Allaert FA, Guovon B, Ferdvnus C, et al. Assessment of the discriminating power of identifiers for record linkage. Rev Epidemiol Sante Publique. 2004; 52:431-40.

4. Karmel R, Gibson D. Event based record linkage in health and aged care services data: a methodological innovation. BMC Health Ser Res. 2007; 7:154-64.

5. Li B, Quan H, Fong A, Lu M. Assessing record linkage between health care and vital statistics databases using deterministic methods. BMC Health Serv Res. 2006; 5; 6: 48-57.

6. Sauleau EA, Paumier JP, Buemi A. Medical record linking in health information systems by approximate string matching and clustering. BMC Medical Informatics and Decision Making. 2005: 5:32-44.

7. Hewitt JB, O'Connor M. Connecting care through EMPIs. J AHIMA. 2002: 73 (10):32-4, 36-8, 2.

8. Arellano MG, Weber GI. Issues in identification and linkage of patient records across an integrated delivery system. J Health Inf Manag. 1998; 12 (3):43-52.

9. Malin B, Sweeney L. A secure protocol to distribute un-linkable health data. AMIA 2005 Symposium Proceedings; 2005. 485.

10. Health Insurance Probability and Accountability Act. Available from: www.hhs.gov/ocr/privacysummary.p df

11. Malin B. An Evaluation of the current state of genomic data privacy protection technology and a roadmap for the future. JAMIA. 2005:12(1):28-34.

12. Johansson La, Westerling $R$. Comparing hospital discharge records with death certificate: can the differences be explained? J Epidemiol Community Health. 2002: 56 (4):301-8.

13. Zingmond DS, Ye Z, Ettner SL, Liu $\mathrm{H}$. Linking hospital discharge and death records - accuracy and source of bias. J Clin Epidemiol. 2004: 57: 21-9.

14. Kwok HK, Steven N. Dynamic patient data bases: The foundation of an integrated approach to outcome for the health care professionals. Med Info.1995; 1:483- 91.

15. Stucki G, Stier-Jarmer M, Grill E, Melvin J. Rationale and principles of early rehabilitation after an acute injury or illness. Disability and Rehabilitation. 2005: 27(7/8):353-9.

16. Cohen J, Bilsen J, Miccinesi G, Lofmark R, Addington -Hall J, Kaasa $S$, et al. Using death certificate data to study place of death in 8 European countries: 
opportunities and weaknesses. BMC Public Health. 2007; 7:28391.

17. Fung V, Cheung NT, Ho E, Cheung $\mathrm{C}$, Chan $\mathrm{H}$, Tsang $\mathrm{K}$, et al. Building a womb-to-tomb health record in Hong Kong: an application of information architecture: Medinfo. 2007; 12(Pt 1):474-7.

18. Sek A, Cheung NT, Choy KM, Wong WN, Tong AY, Fung $\mathrm{VH}$, et al. A territory-wide electronic health record-from concept to practicality: the Hong Kong experience. Medinfo. (2007); 12(Pt 1):293-6.
19. Grannis SJ, Overhage JM, Hui S, McDonald CJ. Analysis of a probabilistic record linkage technique without human review. AMIA Annu Symp Proc. 2003:25963. 
